Horizons philosophiques

\title{
La problématique des langues en Algérie : historique, situation et conséquences
}

\section{Mansour Benchehida}

Volume 12, numéro 1, automne 2001

Langue : identité plurielle

URI : https://id.erudit.org/iderudit/801198ar

DOI : https://doi.org/10.7202/801198ar

Aller au sommaire du numéro

Éditeur(s)

Collège Édouard-Montpetit

ISSN

1181-9227 (imprimé)

1920-2954 (numérique)

Découvrir la revue

Citer cet article

Benchehida, M. (2001). La problématique des langues en Algérie : historique, situation et conséquences. Horizons philosophiques, 12(1), 125-135.

https://doi.org/10.7202/801198ar d'utilisation que vous pouvez consulter en ligne.

https://apropos.erudit.org/fr/usagers/politique-dutilisation/ 


\section{La problématique des langues en Algérie : historique, situation et conséquences}

\section{Introduction}

Au Maghreb, l'idée d'état-nation a vu le jour durant la colonisation et par opposition à un quotidien ressentie pour le moins humiliante par les autochtones. L'Algérie a pris conscience de son existence au fur et à mesure de la lutte contre le colonialisme. Avant l'occupation française et avant même l'avènement du monothéisme, des tribus éparses fonctionnaient selon un schéma autarcique. Elles se présentaient un peu à la manière des cités grecques, avec cependant des différences notables. Des spécificités articulées autour d'une onomastique lorsque le nom du patriarche fondateur se retrouve chez la quasi totalité des familles et quelques exceptions qui représentent des alliés ou des gendres'. II arrive que des noms différents se fédèrent autour de leur coutume commune de nomadisme pour certains, de leur activité agraire ou de leur obédience à une confrérie ${ }^{2}$ pour d'autres. Ceci reste des exceptions qui confirment la règle. Les tribus, en tant qu'entités socio-économiques, étaient très différentes de la cité hellène en ce sens que cette dernière était composée d'une pluralité d'individus unis dans la conscience d'un projet commun audelà du nom ou de la relation familiale. Ce qui n'était pas du tout le cas dans le Maghreb. Cette relation tribale fut renforcée par l'arabité qui accompagna l'Islam, dans ce qu'elle a de primauté de la collectivité et de reniement de l'individu. Elle accentua la solidarité communautaire et installa dans l'imaginaire une conception qui, paradoxalement, émerge encore aujourd'hui dans le quotidien ${ }^{3}$.

1. La règle dans la famille maghrébine qui est patrilinéaire et patriarcale, impose que ce soit la femme qui intègre le clan du mari. Mais, selon une tradition qui sera renforcée par I'Islam, des enfants sans famille ou des orphelins élevés au sein de la communauté deviennent parfois des chefs de familles tout en conservant leur nom d'origine étranger au clan.

2. Un exemple significatif se trouve à Mostaganem. La population rurale des environs de cette ville et qui se reconnaît sous le nom de Medjahers constitue de fait une confédération de vingt-cinq grandes tribus porteuses d'une centaine de patronymes. Ils sont tous disciples d'une même zaouia (confrérie musulmane autour d'un saint local), celle de Betekouk. Cette zaouia "quadrille» la région par cinq marabouts qui ont la particularité d'être frères ou fils les uns des autres.

3. Les conseils de tribus berbères (arouch, plur. de arch : tribu) livre une guerre de nerfs au Pouvoir. Celui-ci leur ayant interdit l'accès de la capitale pour manifester un ras-le-bol général, ils ont, par rétorsion, barré la route d'un sanctuaire de la révolution et empêché le Président de la République et le Gouvernement d'aller s'y recueillir solennellement le 
À travers ces tribus, la diversité des parlers portait sur des variances libres, des prononciations ou des écarts sémantiques limités qui ne gênaient pas la communication sur une aire qui correspondait à l'activité des uns et des autres. Ainsi les berbérophones limitrophes d'arabophones constituaient un tampon linguistique diglossique qui permettait de croire à une uniformité relative de la langue. La culture était en quelque sorte une représentation plus ou moins élaborée de la mythologie tribale et de l'épopée qui se manifestait à travers une geste ${ }^{4}$. Certaines tribus ont eu des poètes de l'oralité qui ont immortalisé les hauts faits de leurs tribus et de leurs régions. Ainsi Mostéfa Benbrahim surnommé le barde des Béni Ameur, Mohamed Belkheir, chantre de la résistance des Ouled Sidi Cheikh, etc. utilisaient un arabe parlé ou un berbère usuel que tous comprenaient plus ou moins 5 . Les différences langagières perceptibles relevaient de l'identité de la tribu. Chaque parler local acceptait facilement la coexistence avec d'autres parlers, censés être la marque des voisins qui étaient parfois des cousins. Dans ce flou, et avec les limites des communications et des déplacements, vivait une population éclatée qui pressentait des points communs, à l'origine d'une vague notion unitaire. Ceci en faisait un peuple sans frontière tranchée ni conscience nette. Ils avaient pour dénominateur commun d'abord le berbère puis la religion musulmane qui eut l'heur de conforter beaucoup de leurs pratiques, de structurer leurs habitudes, d'apporter un justificatif divin à un comportement à peine amendé. Quant à l'idée du monothéisme, elle existait depuis l'expansion du judaïsme, puis de la chrétienté qui s'ensuivit.

L'Islam est venu au Maghreb par l'intermédiaire d'une seule et unique tribu, les Banou Hillal, de la Haute Egypte. La première remarque est que cette tribu elle même n'est pas arabe mais autochtone du pays du Nil, lieu de brassage et de passage et où l'essentiel de la population remonte du sud africain.

Après quelques batailles contre les intrus, les indigènes berbères, policés par les croyances hébraïque et chrétienne déjà répandues,

20 août de cette année. Le Président et l'ensemble des ministres n'ont pu que rebrousser chemin. II s'agit là d'une démonstration de la force et de la cohésion de ce schéma social traditionnel.

4. La geste tribale était magnifiée dans des poésies orales dite "chir melhoun". Répétée par des meddahs (espèces de conteurs itinérants) ou des récitants attitrés. Elle constitue l'essentielle d'une littérature dont la nature non écrite a constitué une mémoire approximative mais vivace contre le projet colonial d'acculturation.

5. "Chir melhoun" n'est pas tel que le croient beaucoup, une poésie rimée. Le mot "lahana" c'est faire une faute, un écart par rapport au classique. "Chir melhoun" veut dire poésie où l'on remarque des écarts grammaticaux par rapport à la norme classique. (Amar Belkhodja, "Le melhoun : un riche parent pauvre" in El Moudjahid, quotidien national du mercredi 27 octobre 1993, rubrique culture p. III). 
montrent une attitude favorable aux idées et à l'idéologie monothéiste. L'essentiel des pratiques et des traditions ancestrales se superpose avec bonheur aux nouvelles idées prônées par la religion de Mohamed ${ }^{6}$. Bien plus tard, quand l'Emir Abdelkader' voulut unifier la résistance, chaque tribu, et de manière indépendante, lui présentait son ralliement. II eut comme alliées presque toutes les tribus du centre et de l'ouest algérien dont plusieurs sont situées dans l'actuel Maroc. C'est dire que d'une part, on peut considérer que ce fut le fondateur de l'état "algérien", mais que d'autre part, on ne doit pas considérer le territoire conquis à sa cause comme configuration naturelle d'une nation potentielle.

La colonisation avait prévu un peuplement définitif d'un territoire estimé vide. Elle s'est donné l'autorité de nier l'existence d'un peuple et les moyens de l'éradiquer. La guerre de libération ${ }^{8}$ contre cette situation extrême a installé une idéologie de guerre sans nuance dans le but d'une mobilisation indispensable à une lutte sans merci.

Ainsi au lendemain de l'indépendance, le nationalisme nourri depuis toujours par un populisme fédérateur, ne fait que conforter des idées à l'emporte-pièce et qui étaient des slogans de combat plutôt que des vérités historiques. Ce fait a été accentué par la nature unanimiste de l'embrigadement des masses indispensables quand on veut remettre en cause la présence plus que centenaire de colons en Algérie. Une fois l'état de grâce de la nouvelle souveraineté passé, la problématique langue-identité-culture s'est posée dans une complexité que seule pouvait résoudre une communication accomplie et un système d'information (médias) et de formation (système scolaire) serein. Or d'autres priorités occupaient les décideurs. Entre les tenants de la «modernité» et les partisans de «'authenticité"9, les prises de positions se sont exacerbées. Une écriture sereine de l'Histoire ${ }^{10}$ reste à faire et n'est pas envisageable dans la situation en cours.

6. Lire un roman informé et significatif à ce sujet : Driss Chraïbi, La mère du printemps, Seuil, Paris, 1982.

7. L'Emir Abd-El-Kader (1808-1883) est considéré comme le fondateur du premier état Algérien. II a frappé une monnaie et érigé une capitale mobile (faite d'une cohorte de tentes). II lutta contre la colonisation et eut des succès militaires reconnus avant de céder après un demi siècle de résistance.

8. N'ayant jamais cessé sous une forme sporadique, elle devient guerre totale entre 1954 et 1962.

9. "Tous les musulmans de langue arabe s'estimaient Arabes», Maxime Rodinson, Les Arabes, PUF, Paris 1979 p. 32.

10. «L'Histoire est aussi un phénomène culturel dans le sens où ce qui importe pour assurer la cohésion d'un peuple comme de tout groupement, c'est moins l'ensemble des événements dans sa réalité objective que l'image, socialement élaborée et transmise, qu'en garde le groupe". Maxime Rodinson, Les Arabes, PUF, Paris, 1979 p. 20. 


\section{La culture :}

Les définitions dans ce domaine abondent, nous nous tiendrons aux plus généralistes. La culture procède des croyances d'une population; c'est un ensemble de mythes, de rites, de coutumes, de pratiques qui constituent des points communs et donc des repères.

En Algérie, à l'aube de l'indépendance, beaucoup de comportements présentaient le caractère serein de la tradition sans prétention, prêts à négocier la modernité qui se présentait comme un volet du progrès et un atout de la liberté. Avec les tiraillements du pouvoir, la malvie, terreau prolifique des extrémismes, et les énormes erreurs de gestion du pays, l'islamisme a mis en place sournoisement une multitude de gestes, d'attitudes, de parlers qui s'incrustent dans le quotidien. Ce quotidien se fascise lentement mais sûrement ${ }^{11}$. Les attitudes se radicalisant, l'écart culturel entre ceux qui se pensent arabes et ceux qui sont berbères devient évident. Les berbères alors remettent violemment en avant leur culture $^{12}$ tout en se prévalant de revendications citoyennes et républicaines, la littérature raconte les mythes et les légendes d'un terroir à peine mâtiné de monothéisme ${ }^{13}$, le théâtre quoique moribond, pour différentes raisons entre autres la langue de communication, réintroduit l'oralité 14 ainsi que ses vecteurs de transmission. La culture qui par essence doit être en constante négociation avec l'actualité, la modernité, par rapport au terroir, se retrouve bousculée par des injonctions religieuses de type terroriste. A contre courant s'impose dans la chanson, le "raï», sorte d'expression du spleen et de la misère de la jeunesse. Cette musique bafoue les idées reçues concernant la langue, les comportements. Elle marque surtout une révolte spontanée d'une jeunesse qui s'estime trompée sur son avenir, dans sa formation, dans sa culture. Cette musique a émergé grâce aux moyens de communication. Elle a été consacrée en France avant d'être consentie en Algérie. Elle est l'incarnation la plus forte d'une culture faite dans la désarticulation, dans la rue et dans une constante opposition au pouvoir, aux bastions du savoir bien

11. Par exemple les femmes ne portent plus le voile blanc typiquement algérien haïk. Elles ont opté lentement et radicalement vers le hidjab, vêtement étranger à la culture maghrébine et qui pourtant se rencontre systématiquement dans les rues.

12. Les revendications berbères ont pris un aspect public avec le printemps 1980 qu'on a nommé "le printemps berbère". Depuis, les revendications sont périodiques, elle se focalisent autour de la corruption généralisée, d'un déni des droits omniprésent dans le quotidien.

13. Lire les œuvres de Rachid Boudjedra, de Rachid Mimouni, en particulier La malédiction, Stock, Paris 1993, de Assia Djebar, Vaste et ma prison, Albin Michel, Paris, 1997.

14. Utilisation du meddah (espèce d'aède de la tradition orale) dans le théâtre de Alloula Abdelkader, assassiné par les islamistes) et de Ould Abderrahmane Kaki notamment. 
pensant (université, école coranique ${ }^{15}$ ). Elle fait l'objet de tentative de récupération du Pouvoir et en même temps demeure cible constante de la vindicte islamique.

Le quotidien a été longtemps un ensemble de pratiques hérité de la tradition. Cette culture tenait d'un fond tantôt à prépondérance berbère, tantôt d'us relevant de l'arabité. Dans les années 80 , le président Boumediene disparaît, homme Cromwellien ${ }^{16}$ utilisant un pouvoir fort et sans partage. Les différents clans s'affrontèrent alors pour le pouvoir. La lutte fut discrète, imperceptible pour le large public, du fait que les affrontements sans merci se déroulaient au sein du Congrès du Parti unique. Joute de sérail, puisque régnait la pensée unique se prévalant de la raison d'état, toute contradiction évacuée. Le consensus se fit autour de l'homme jugé le plus faible, le plus maniable du milieu initié, Chadli Bendjedid. Chaque pôle d'influence espérait en faire sa créature. II déverrouilla, brusquement et sans étape, non seulement le champ politique qui explosa en réaction à la coercition qu'il vivait, mais également les mécanismes économiques peu compétitifs, vétustes et rentiers. Cependant, sa faiblesse permit à la corruption, jusqu'alors, confinée au niveau du cercle restreint des familiers de Boumediene, d'apparaître progressivement à tous les niveaux. Elle prit bientôt des allures de banalité et se généralisa. Dès lors, s'installa dans une fracture sociale de plus en plus béante, la crise. Une déstabilisation, résultant de la brusque transformation d'une économie socialisante et seule expérience sociale des masse, accentua un équilibre factice parce que s'appuyant sur des slogans unanimistes. Une économie de marché faite de réalités sans demiteinte, prit forme de manière chaotique et surtout en se cherchant des bases juridiques adaptées, ce qui n'existait pas. Face à une corruption qui devint publique et généralisée, l'explosion populaire eut lieu en octobre 88. Elle permit aux idées fondamentalistes de se manifester et surtout de récupérer le mécontentement populaire. Parée de l'innocence religieuse et se prévalant du divin, l'adhésion fut foudroyante pour un pouvoir qui s'y trompa une fois de plus. II adopta une position louvoyante car il voyait dans «la tendance religieuse» un moyen de contenir les idées de gauche et de démocratie moderniste. Le fondamentalisme s'immisça dans les pratiques, les habitudes vestimentaires, les relations les plus anodines ${ }^{17}$.

\section{Appelées Zaouia.}

16. En ce sens qu'il prônait un nationalisme farouche et exigeait de lui-même et des autres un ascétisme exemplaire pour le peuple dont il fabriquait le bonheur (plein emploi, socialisme réel) sans tenir compte de son avis, marque d'un mépris qui contribuera à la violence qui s'ensuivit.

17. Ainsi le salut populaire qui était "bonjour" (sabah el kheir) disparut au profit d'un farouche "que la paix soit avec vous" (salam laykoum). Ce qu'on oublie c'est que cette 
Tout s'imprégna d'une culture d'importation ${ }^{18}$ qui est toujours en train de bousculer la culture traditionnelle, naturellement exubérante et méditerranéenne, avec de plus en plus de férocité et de fascisme ${ }^{19}$.

\section{L'identité}

D'une langue problématique et d'une culture aux abois, il résulte une identité disloquée. Mais la difficulté identitaire reste aussi et surtout la conséquence de l'absence d'un projet de société. Les différentes tendances, se relayant à l'ombre d'un Pouvoir dont la finalité est le pouvoir, les lois semblent se contredire, voire s'abroger les unes les autres. La manipulation, souvent elle-même manipulée, contribue à une confusion entretenue. L'école, dont les textes se sont arrêtés à la formation d'un militant socialiste et musulman 20 des années de Boumédiène, n'arrive plus à remplir sa fonction. S'agit-il de focaliser des compétences et des volontés autour d'un projet social, indépendamment des croyances politiques, religieuses, en reléguant la question ethnique au rang de mythe, ce qu'elle n'aurait pas dû quitter? S'agit-il d'asseoir une population autour d'un dénominateur commun et fédérateur, la religion? D'une position à l'autre, l'opposition est diamétrale et les passions à l'ordre du jour. Dans un cas, la centralité revient à la république et dans l'autre, l'état s'estompe dans une vastitude musulmane ${ }^{21}$ qui dépasse les nationalités 22 .

formule relève d'un état de suspicion générale au temps du Prophète et qu'elle engageait le locuteur et l'interlocuteur qui y répondait à une position pacifiste; la nonréponse est en soi, hostilité dangereuse. Son adoption installe de facto une relation d'agressivité ou de soutien, on est décidément loin de l'idée de société citoyenne.

18. La pratique du "siouak", bout d'écorce d'un arbre qui pousse en Inde, le port d'un type de pantalon (dit "salafiste" et qui de ce fait est indice de reconnaissance entre islamistes) qui n'a rien à voir avec le sarouel musulman traditionnel, la manière de saluer, les accolades.

19. Au début d'août 2001, un quartier de Hasi Messaoud, habitée surtout par des femmes travailleuses chez les pétroliers, fut envahi. Des dizaines de femmes furent violées, torturées et tuées par des jeunes nervis poussés par les prêches enflammés d'un imam local. II y a des dizaines d'exemples analogues. II suffit de lire la presse.

20. La loi 76-35 du 16 avril 1976 portant sur l'École fondamentale institue un système éducationnel basé sur une pensée politique nationaliste et socialiste, sans nuance et sans état d'âme. II est renforcé par le rectificatif de l'ordonnance $n^{\circ} 52$ du 29/6/76 qui impose "une éducation islamique".

21. Appelée "Ouma». Ce terme désigne tous les pays musulmans sans limite de frontières (non reconnues dans la vision musulmane) ni de nationalités (la qualité de musulman l'annihile).

22. "Le peuple arabe actuel (est) composé en grande partie d'arabisés plus ou moins récents, même si, dans beaucoup de cas, ils croient descendre des Arabes d'Arabie", Maxime Rodinson, Les Arabes, PUF, Paris, 1979 p. 22. 
Quoique la donne islamique dans l'identité algérienne est incontestable, il y a des Algériens qui ne s'y reconnaissent pas ${ }^{23}$ ou ne s'y reconnaissent que partiellement ${ }^{24}$. Les Algériens ont tendance à se déclarer "Arabes» avec conviction. Ceci se comprend aisément quand on relit l'Histoire tourmentée de la région. Mais la grande question est : estce que les arabes incontestables, ceux d'Orient, en particulier de l'Arabie Saoudite, acceptent les maghrébins en tant qu'Arabes? Pour ma part à chaque fois qu'un Algérien de mes connaissances se prépare à aller en pèlerinage en Arabie, je ne manque pas de lui demander de questionner les autochtones sur notre "race». Je m'amuse systématiquement des réponses : pour ces Arabes, nous sommes ce que nous voulons, peutêtre des roums ${ }^{25}$, jamais, au grand jamais, des Arabes. Et ils ont raison. Mais notre réseau informatif et de formation n'arrive point à engager la discussion là-dessus et notre Histoire reste à réécrire.

\section{La langue :}

La langue berbère a dû être celle de toute l'Afrique septentrionale, de la Libye à l'Atlantique marocain en passant par le Yémen. Elle provient du phénicien qui se transforma en lybique puis en plusieurs dialectes berbères. Il y eut par la suite différentes langues qui s'installèrent dans le sillage des pouvoirs politiques. C'est le cas du latin des Romains, de l'Arabe véhiculant l'Islam, du Turc des Ottomans, du français enfin. Le peuple s'est arabisé pour les besoins de cette religion dans laquelle il se reconnaissait. II a soit laissé tomber en désuétude des langues qui n'ont concerné que les élites comme le latin, les clients du pouvoir comme le turc, soit ignoré une langue qui lui a semblé contre ses croyances du moment, comme le français. L'arabe s'est maintenu sous forme de dialecte où on relève des formes adaptées ${ }^{26}$. La langue arabe est loin d'être flexible du fait de la norme. Sa seule norme se veut éternelle, sans amendement du moins dans sa syntaxe, car elle se réfère au Coran, parole divine.

23. Il y a en Algérie une faible minorité chrétienne (évaluée à 300000 en 1982, elle serait moins importante aujourd'hui), elle est dirigée par une structure cléricale bel et bien existante. II y a aussi des intellectuels qui se disent (avec discrétion) athées.

24. La qualité de musulman concerne le non-pratiquant, et toute la gamme de ceux qui sans être des inconditionnels se disent musulmans.

25. C'est le nom attribué aux byzantins par les Orientaux et d'une manière général aux européens par les Algériens de l'ouest.

26. Dans l'onomastique, on relève des prénoms comme Miloud à l'ouest algérien qui étonne les orientaux qui ne connaissent que Mouloud, comme Harrag (le brûleur, le pyromane) qui est blasphème dans l'orthodoxie musulmane et met dans l'embarras ceux qui le portent, ce qui n'était nullement le cas dans les années 90 . 
À travers ces différentes situations chaque langue a été bien sûr un vecteur de communication, mais aussi souvent un facteur de démarcation, de rupture. Si le phénicien et le lybique ont été des outils de commerce entre des étrangers débarquant et des autochtones, ils ont été aussi un moyen de communication entre les tribus, un espace de qualification et de quantification des gestes d'un quotidien qui se donne par là un dénominateur commun. Ainsi se profile une communauté embryonnaire; une perception qui structure l'imaginaire autour d'une notion d'unité diffuse, un peuple éclaté en somme.

En parallèle, la langue a existé dans une dimension de particularisme. Elle a été un critère de différenciation entre ceux qui étaient là et ceux qui arrivaient. Ce schéma est aussi valable pour ceux qui ont vu débarquer les Phéniciens de Didon que ceux qui ont accueilli les janissaires Turcs ou subi l'assaut de la marine Française.

Lieu de communication, espace de communion mais aussi critère d'identification, indice de délimitation, la langue en Algérie est un corpus où se décèlent toutes les invasions. Ainsi toutes les différentes langues des différentes périodes se retrouvent à un niveau ou un autre, dans le parler d'une région plutôt que dans celle de l'autre. L'arabe dialectal s'est maintenu grâce au concept qui le porte et le consacre : l'Islam. Le berbère a survécu parce qu'il a été originellement général et qu'il s'est réfugié dans les sommets rébarbatifs et les recoins isolés. Le français parce qu'il a été porté par le colonialisme conquérant, mais aussi par la modernité qu'il est censé incarner. Longtemps les trois langues ont coexisté, en tant que vecteurs d'échanges et dans leurs espaces distincts.

Systématiquement, pour parer au colonialisme et afin de donner une façade unanimiste, la langue arabe, porteur de lidée ségrégative forte de la religion, a été préférée. Le berbère, langue d'une partie de la population a été "oublié" presque avec l'assentiment des berbérophones, premiers partisans de la Cause de l'indépendance. Le phénomène a accompagné les différentes révoltes qui ont jalonné la deuxième moitié du XIXe siècle. Puis sont venues les revendications suscitées par les autochtones qui ont eu l'occasion de voir "'ailleurs" grâce à leur mobilisation militaire pour les deux guerres mondiales. Durant la guerre de libération, on utilisa la langue française pour toute sorte de document ${ }^{27}$ destiné à l'opinion internationale. L'activité auprès des masses, les mots d'ordre, de propagande, les chants et les slogans de mobilisation étaient en arabe dialectal, compris par tous. Après l'indépendance, le problème de la langue s'articula

27. La déclaration du $1^{\text {er }}$ novembre 1954, acte de naissance de l'insurrection armée, est rédigé en français. 
autour du français, langue vernaculaire, et de l'arabe, marque identitaire différenciée. Au niveau de la guerre, puis au stade de la reconstruction du pays, se déploie encore l'ambivalence des langues : communication et opposition.

À partir de 1965, les berbères rappellent timidement leur différence linguistique. Mal leur en prit, face aux revendications de ce qui est devenu officiellement une minorité linguistique menaçante pour la cohésion et la stabilité; l'armée, seul ensemble structuré, s'est faite force publique contre le “désordre». La répression du pouvoir de l'époque 28 a posé sur la berbérité une chape de plomb pour des décennies. Le français enseigné majoritairement à l'école commence à céder la place à une arabisation galopante rendue incontournable par une mesure de rétorsion de l'expuissance coloniale à ne pas fournir assez d'enseignants de français à une école algérienne au désarroi29. Cette arabisation fut encouragée par une majorité de rapatriés qui, durant les années de braise, s'étaient réfugiés dans les pays voisins ou étudiaient dans les universités de l'Orient. Beaucoup sont revenus avec des diplômes mais aussi avec des idéologies panarabiques et panislamiques plus ou moins avouées. Ils avaient des diplômes et qui plus est, les démarquaient du français assimilé à la puissance coloniale honnie. Ils s'installèrent aux commandes et à la périphérie du pouvoir. Ils jouissaient d'une confiance qui faisait défaut aux compétences produites par l'école française ${ }^{30}$. Ces données et d'autres plus nuancées mais qui participent de la même logique ont installé un extrémisme linguistique qui vit d'amalgames : l'arabe doit être généralisé à tous sans exception, nous sommes tous des arabes, l'arabe est une langue sacrée ${ }^{31}$, etc.

Actuellement, avec la mondialisation incarnée par les canaux de télévisions satellitaires, le français se réinstalle. Il est toutefois mis à l'index par une majorité de jeunes, formés à «l'école fondamentale», qui y voit un danger pour son avenir professionnel donc substantiel. II y a lieu de remarquer qu'est couramment admis en privé l'ineptie d'un

28. Le premier président de l'Algérie indépendante, Ahmed Benbella a tenu les commandes de 1962 à 1965 d'un pays en liesse. II fut renversé par Boumédiène, partisan de l'ordre musclé et d'un socialisme sans contestation. Les répressions des régions kabyles furent alors sans demi-mesure.

29. Voir mémoire rédigé par Mostéfa Lacheraf, Ministre de l'Éducation des années 1970.

30. Allusion à “la promotion Lacoste", loi du même nom qui, à partir de 1958 , favorisa la formation et l'intégration d'Algériens aux postes intermédiaires.

31. "La langue arabe comme la langue de la révélation et, en somme, comme la langue de Dieu» (p. 20); "Le Coran, parole de Dieu, est en langue arabe de sorte que les musulmans considèrent en somme l'arabe comme la langue de Dieu." (p. 36), Maxime Rodinson, Les Arabes, PUF, Paris, 1979. 
monolinguisme ringard. La jeunesse, écrasante majorité, a été arabisée dans les pires conditions et cède à une schizophrénie effarante qui fait du français, une langue anti-islamique, impie, donc à proscrire, mais aussi une langue admirée secrètement car passeport intellectuel sans pareil et atout d'un exil en Europe que la jeunesse entrevoit comme seule porte de sortie.

\section{Conclusion :}

La triade culture-identité-langue constitue une même problématique, tant chaque notion est imbriquée dans l'autre. Quand l'une est incertaine, tout l'édifice s'en ressent.

La langue en tant que signifiant de la culture et de l'identité, en tant qu'outil de signification de ces deux concepts, reste un espace d'union et un indice de différenciation. C'est le moyen de communiquer, de se comprendre mais aussi un critère de définition de l'autre, l'étranger. Lorsque cet étranger est aussi indigène que celui qui le désigne, il en résulte une situation potentiellement conflictuelle. Elle peut être suivie d'affrontements. En Algérie, aucune langue n'est admise par tous. C'est un grave dilemme qui se règle au fil des discussions et des leviers prévus par la démocratie. Lorsque celle-ci est absente ou sans tradition, c'est le dérapage. Un dérapage d'autant plus grave que la langue reste une manière de voir, de saisir la difficulté, un outil de solution. Cependant chaque langue inculque à son utilisateur une vision qui peut s'avérer incompatible avec le vis-à-vis. Surtout si la passion exacerbe les points de vue.

Dans le cas de l'Algérie, c'est un pays récent politiquement, jeune socialement et dont le passé tumultueux a installé des idées préconçues. Ces contrevérités ayant arrangé un système par essence populiste, on n'a jamais pu écrire l'Histoire malgré les innombrables commissions et fondations ${ }^{32}$, on n'a jamais pu décider d'un système éducatif en dépit de farouches volontés ${ }^{33}$ qui émergent de temps à autre, on n'a jamais pu convenir d'un projet de société admis par la plupart et dans une clarté voulue par tous. Avec le timide début d'une démocratie qui s'est installée, sous forme de pluralisme politique et qui a permis aux islamistes de pré-

32. II y a une multitude de fondations et d'associations nationales ou locales qui s'occupent de réécrire l'histoire. Aucune n'a abouti à des résultats publiables à ce jour.

33. Dont la dernière émane de l'actuel président, Bouteflika. Installée en 1999 avec beaucoup de moyens et l'échéance d'une année puisque l'urgence est indéniable. Après des travaux houleux entre modernistes et conservateurs, le rapport terminé et remis à la Présidence en mai 2000 n'est toujours pas connu. 
tendre au pouvoir en se prévalant du jeu démocratique qu'ils promettent d'éradiquer dés que possible, les positions se sont radicalisées et le bon sens a disparu. II ne reste que le temps et la lassitude pour élaborer lentement notre identité et asseoir une langue qui soit essentiellement vecteur de communion, malgré nous. Mais cela demande des décennies de guerres civiles, de tragédies sociales, de combats fratricides. Nous y sommes déjà en quelque sorte.

\author{
Mansour Benchehida \\ Professeur \\ Université de Mostaganem
}

